\title{
Pressure and velocity in intraocular and subarachnoid space fluid chambers: an inseparable couple
}

\author{
Hanspeter E. Killer ${ }^{1,2} \cdot$ Ruow Hou ${ }^{3} \cdot$ Peter Wostyn ${ }^{4} \cdot$ Peter Meyer ${ }^{2,5} \cdot$ Achmed Pircher $^{1,6}$
}

Received: 25 August 2018 / Accepted: 17 September 2018 / Published online: 12 October 2018

(c) The Royal College of Ophthalmologists 2018

Primary open-angle glaucoma (POAG) is a leading cause for irreversible blindness in the world. The risk factor most frequently associated with POAG is increased intraocular pressure (IOP), for a long time considered to be the sole cause for glaucomatous optic nerve damage. It was thought that pressure, a non-directional scalar force, causes direct mechanical damage to the retinal ganglion cells and their axons, leading to cupping of the optic disc and visual field loss.

The concept of raised IOP as the single cause for optic disc excavation and visual field loss came under scrutiny when larger studies on glaucoma patients found glaucomatous disc excavation and glaucomatous visual field defects also in patients with a "statistically" normal IOP. A new term, normal tension glaucoma (NTG), is now used to describe this condition. Once hardly recognized, glaucomatous optic disc changes are found in up to $40 \%$ of POAG patients with IOP $<21 \mathrm{mmHg}$ in the western hemisphere [1] and for up to $90 \%$ of POAG in the Far East [2].

Such observations have stimulated researchers to find alternative pathophysiological mechanisms that could explain glaucomatous ON damage in patients with "normal" IOP [3]. One direction of the research focused on a

Hanspeter E. Killer

profesrielkiller@gmail.com

1 Department of Ophthalmology, Kantonsspital Aarau, Aarau, Switzerland

2 Department of Ophthalmology, University Hospital Basel, Basel, Switzerland

3 Department of Neurosurgery, Beijing Tongren Hospital, Capital Medical University, Beijing, China

4 Department of Psychiatry, PC Sint-Amandus, Beernem, Belgium

5 Department of Biomedicine, Ocular Pharmacology and Physiology, University Hospital Basel, Basel, Switzerland

6 Department of Neuroscience/Ophthalmology, Uppsala University, Uppsala, Sweden vascular component [4]. In some patients vascular dysregulation was indeed demonstrated to be present in NTG [4]. Recent research has brought pressure back into the ball park by postulating a disbalance between IOP and intracranial cerebrospinal fluid pressure (ICP) at the level of the lamina cribrosa. The so-called trans-lamina cribrosa pressure difference (TLCPD) is defined as the intraocular minus the intracranial pressure (IOP - ICP) [5]. The advocates of this concept suggest that this pressure gradient causes shearing forces at the lamina cribrosa that damage the axons at that site due to an abnormally high-pressure gradient between the intraocular and the retrobulbar "chambers" [5].

Intraocular pressure results from aqueous humor (AH) in the anterior chamber just as pressure in the brain and the pressure in the spinal cord, and in the subarachnoid space of the optic nerve results from cerebrospinal fluid (CSF). Recent research of CSF composition and CSF flow dynamics in patients with NTG has demonstrated impaired CSF turnover in the orbital subarachnoid space is most pronounced in the bulbar segment of the optic nerve portion, the region right behind the lamina cribrosa [6]. This constellation is now referred to as "optic nerve sheath compartment syndrome" [7]. It has been described in a subgroup of patients with papilledema, as well as in patients with NTG [6,8].

Granted that the ICP is elevated in patients with papilledema and that the CSF pathway from intracranial to orbital CSF space is patent, papilledema evolves due to blockage of axoplasmic transport caused by pressure elevation. At the same time, the velocity of CSF is reduced according to Bernoullis equation $\rho \frac{v^{2}}{2}+p=c$ (where $\rho$ is the density of the fluid, $v$ velocity of the fluid, $p$ pressure, and $c$ constant). Reduced CSF velocity results in the reduced CSF turnover in the subarachnoid space of the optic nerve, which consequently diminishes clearance of peptides and proteins in the environment surrounding the optic nerve. Sampling of a CSF specific protein, L-PGDS (prostaglandin synthase) demonstrated high locally elevated concentrations in the subarachnoid space surrounding the 
optic nerve in patients with papilledema, and to some extent in patients with NTG [9]. Elevated L-PGD concentrations in CSF resulted in a reduction of astrocyte proliferation and in a markedly reduced adenosine triphosphate production [10]. Further, studies using computer-assisted cisternography demonstrated reduced contrast density in the orbital portion of patients with elevated ICP [8], as well as in patients with NTG [6]. In addition, reduced CSF velocity in the subarachnoid space of optic nerves in patients with NTG was confirmed with a Stejskal-Tanner monopolar diffusion sequence on MRI, a new noninvasive method for the study of CSF dynamics [11]. As local pressure measurements in the subarachnoid space of the optic nerve are not possible in a clinical setting, the diameter of the optic nerve sheath can serve as an indicator of pressure. Watanabe [12] showed that the optic nerve sheath diameter increases with increasing ICP. Studies of the optic nerve sheath diameter in NTG patients rendered conflicting results between a Chinese and a European population. While the optic nerve sheath diameter was reduced in the Chinese population [13], it was enlarged in the European cohort [14, 15]. Both findings, however, indicate a disturbance of CSF dynamics in the orbital subarachnoid space. The disparity might be explained by genetic differences or by the fact that the European population consisted of more advanced glaucoma cases. From the latter point of view, it has been suggested that a conversion of the orbital CSF pressure from low to high may occur during the disease process of NTG. During the early stages of NTG, before the development of compartmentation, CSF may communicate freely between the intracranial subarachnoid space and the subarachnoid space of the optic nerve. At this stage, the low lumbar CSF pressure reported in NTG patients may correspond to a low orbital CSF pressure, resulting in a narrower orbital optic nerve subarachnoid space [16]. In more advanced stages of NTG, the CSF pressure in the subarachnoid space of the optic nerve may convert to high CSF pressure due to compartmentation and the absence of free communication between the intracranial subarachnoid space and the subarachnoid space of the optic nerve. Interestingly, in accordance with this view, Killer and Jaggi [15] noted that the NTG patients in their study represented end-stage glaucoma with progressive loss of visual field.

Unlike the subarachnoid space of the optic nerve that can undergo compartmentation, the fluid-filled chambers of the eye, like the anterior and posterior chambers and the vitreous chamber, are a priori quasi compartments due to their anatomical structure and function. The anterior and posterior chambers are filled with $\mathrm{AH}$, a clear fluid that is constantly produced by the ciliary body with a production rate of $2.5 \mu \mathrm{l} / \mathrm{min}$ [17]. From the ciliary body $\mathrm{AH}$ is secreted into the posterior chamber, from where it flows into the anterior chamber, as well as in the direction of the vitreous cavity. $\mathrm{AH}$ is essential for maintaining a stable anterior chamber and IOP [18]. Its composition has a similarity to blood serum [19]. AH provides nutrition for the corneal endothelium and the lens. It also works as a transport medium that clears potentially harmful metabolic components from the anterior chamber [20]. Proteomic studies have allowed the identification of 676 proteins in the $\mathrm{AH}$; among them signaling proteins, catalytic proteins, complement, enzymes, transport proteins, and structural proteins were identified [21].

The main outflow route of $\mathrm{AH}$ from the anterior chamber is directed toward the trabecular meshwork, from there into Schlemms canal, and then into collector channels that are connected to the intrascleral venous plexus [22]. The anterior chamber, however, is not a completely isolated fluid compartment, as $\mathrm{AH}$ can flow into the posterior chamber and from there into the vitreous cavity, reaching the retinal axons and ganglion cells.

\section{Pressure and velocity-an inseparable couple in fluids}

Pressure is a scalar- and therefore nondirectional force ( $P=F / A$, where $P$ is the pressure, $F$ force, and $A$ area). The force in the equation is caused by the amount of $\mathrm{AH}$ in the anterior chamber and by the constant influx of newly produced AH from the ciliary body. It becomes somehow more complicated when we consider pressure in fluids. It is evident from the Bernoulli and the Navier-Stokes equation that pressure cannot be viewed in isolation when considering a closed-circuit fluid system.

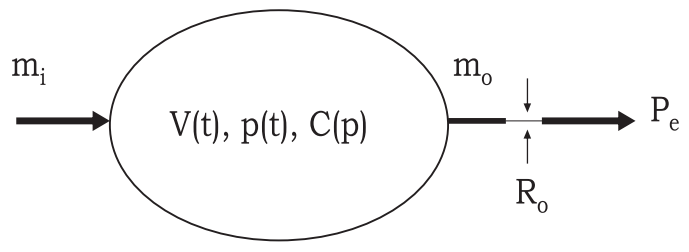

$$
\begin{array}{r}
\partial p / \partial t+\nabla(p v)=0 . \text { Therefore }: \rho(\partial v / \partial t+\nabla v)=-\nabla p \\
+\nabla T+f
\end{array}
$$

where $m_{\mathrm{i}}, V, p, C, m_{\mathrm{o}}, R_{\mathrm{o}}$, and $P_{\mathrm{e}}$ are variables, $V$ is the speed of flow, $\rho$ density, $T$ stress tensor, $p$ pressure, $f$ body force, and $\nabla$ Nabla operator.

\section{Relationship between volume and pressure}

$C=\mathrm{d} V / \mathrm{d} p$. Description of a single-fluid compartment: $\mathrm{C}(\mathrm{p})$ $\mathrm{d} p / \mathrm{d} t=1 / \rho\left[m_{\mathrm{i}}(t)-m_{\mathrm{o}}(t)\right]=$ mass flow, $\rho=$ fluid density 
(incompressible). If influx is constant (mi), then outflux is $m \mathrm{o}=1 / R_{\mathrm{o}}\left[p(t)-p_{\mathrm{e}}(t)\right]$

$P+p h g+\frac{1}{2} p v^{2}=c \cdot \rho \frac{v^{2}}{2}+p=c$

where $v$ is the fluid flow speed at a point on a streamline, $g$ acceleration due to gravity, $z$ elevation of the point above a reference plane, with the positive $z$-direction pointing upward-so in the direction opposite to the gravitational acceleration, $p$ pressure at the chosen point, and $\rho$ density of the fluid at all points in the fluid.

These equations show that pressure and velocity are coupled, as a part of one equation. Pressure and velocity are interdependent. Focusing on one term distorts the assertion of the formula. Changing the pressure will affect the velocity and vice versa. A high pressure therefore results in a lower velocity and vice versa.

Several studies propose that a reduced CSF turnover is associated with neurodegenerative diseases due to toxic agents in a stagnant CSF [23]. Accumulation of certain substances such as beta-amyloid, tau protein, and alphasynuclein is associated with the pathogenesis of neurodegenerative diseases such as Alzheimer's and Parkinson's disease [23, 24]. Amyloid-beta and tau protein, however, are not limited to CSF and brain tissue as several studies have demonstrated amyloid-beta and tau protein in the vitreous body [25]. Its role there is still unknown.

$\mathrm{AH}$, like CSF, needs to be recycled in order to keep the microenvironment of the optic nerve, the anterior chamber, as well as the vitreous cavity intact. Elevated IOP therefore not only harms retinal ganglion cells and axons via a mechanical damage, but also reduces $\mathrm{AH}$ clearing. Lowering IOP therefore means likewise improving $\mathrm{AH}$ circulation, thereby acting neuroprotectively.

\section{Conclusion}

Blood, CSF, and AH undergo constant renewal and clearing processes. Stasis of these fluids leads to malfunction of the tissues supplied by them. Elevated pressure is coupled with reduced velocity and thereby diminished fluid dynamics. Besides causing mechanical damage to tissue, elevated IOP might act as a primary messenger that initiates a reduced $\mathrm{AH}$ clearance that can cause toxic damage. Lowering IOP therefore helps to improve $\mathrm{AH}$ dynamics.

\section{Compliance with ethical standards}

Conflict of interest The authors declare that they have no conflict of interest.

\section{References}

1. Bonomi L, Marchini G, Marraffa M, Bernardi P, De Franco I, Perfetti S, et al. Prevalence of glaucoma and intraocular pressure distribution in a defined population. The Egna-Neumarkt Study. Ophthalmology. 1998;105:209-15.

2. Cho HK, Kee C. Population-based glaucoma prevalence studies in Asians. Surv Ophthalmol. 2014;59:434-47.

3. Killer HE, Pircher. A normal tension glaucoma: review of current understanding and mechanisms of the pathogenesis. Eye (Lond). 2018;32:924-30.

4. Flammer J, Orgul S, Costa VP, Orzalesi N, Krieglstein GK, Serra LM, et al. The impact of ocular blood flow in glaucoma. Prog Retin Eye Res. 2002;21:359-93.

5. Jonas JB, Wang N, Yang D.Translamina cribrosa pressure difference as potential element in the pathogenesis of glaucomatous optic neuropathy. Asia Pac J Ophthalmol (Phila). 2016;5:5-10.

6. Pircher A, Montali M, Wostyn P, Pircher J, Berberat J, Remonda $\mathrm{L}$, et al. Impaired cerebrospinal fluid dynamics along the entire optic nerve in normal-tension glaucoma. Acta Ophthalmol. 2018;96.

7. Killer HE, Subramanian PS. Compartmentalized cerebrospinal fluid. Int Ophthalmol Clin. 2014;54:95-102.

8. Pircher A, Montali M, Pircher J, Berberat J, Remonda L, Killer HE. Perioptic cerebrospinal fluid dynamics in idiopathic intracranial hypertension. Front Neurol. 2018;9:506.

9. Killer HE, Jaggi GP, Flammer J, Miller NR, Huber AR. The optic nerve: a new window into cerebrospinal fluid composition? Brain. 2006;129(Pt 4):1027-30.

10. Xin X, Huber A, Meyer P, Flammer J, Neutzner A, Miller NR, et al. L-PGDS (betatrace protein) inhibits astrocyte proliferation and mitochondrial ATP production in vitro. J Mol Neurosci. 2009;39:366-71.

11. Boye D, Montali M, Miller NR, Pircher A, Gruber P, Killer HE, et al. Flow dynamics of cerebrospinal fluid between the intracranial cavity and the subarachnoid space of the optic nerve measured with a diffusion magnetic resonance imaging sequence in patients with normal tension glaucoma. Clin Exp Ophthalmol. 2018;46:511-8.

12. Watanabe A, Kinouchi H, Horikoshi T, Uchida M, Ishigame K. Effect of intracranial pressure on the diameter of the optic nerve sheath. J Neurosurg. 2008;109:255-8.

13. Wang N, Xie X, Yang D, Xian J, Li Y, Ren R, et al. Orbital cerebrospinal fluid space in glaucoma: the Beijing intracranial and intraocular pressure (iCOP) study. Ophthalmology. 2012;119:2065-73.e1.

14. Pircher A, Montali M, Berberat J, Remonda L, Killer HE. Relationship between the optic nerve sheath diameter and lumbar cerebrospinal fluid pressure in patients with normal tension glaucoma. Eye (Lond). 2017;31:1365-72.

15. Jaggi GP, Miller NR, Flammer J, Weinreb RN, Remonda L, Killer HE. Optic nerve sheath diameter in normal-tension glaucoma patients. Br J Ophthalmol. 2012;96:53-6.

16. Wostyn P, De Groot V, Van Dam D, Audenaert K, De Deyn PP. A general decline in cerebrospinal fluid flow and optic nerve compartmentation: are these sequential steps leading to toxicity in normal-tension glaucoma? Acta Ophthalmol. 2016;94:e242-3.

17. Maus TL, Brubaker RF. Measurement of aqueous flow by fluorophotometry. In: Ritch R, Shielsds MB, Krupin T, editors. The glaucomas. 2nd ed. St Louis: CV Mosbz Co. 1996. p. 447-54.

18. Brubaker RF. Goldmann's equation and clinical measures of aqueous dynamics. Exp Eye Res. 2004;78:633-7.

19. De Berardinis E, Tieri O, Polzella A, Iuglio N. The chemical composition of the human aqueous humour in normal and pathological conditions. Exp Eye Res. 1965;4:179-86. 
20. Huang AS, Francis BA, Weinreb RN. Structural and functional imaging of aqueous humour outflow: a review. Clin Exp Ophthalmol. 2018;46:158-68.

21. Chowdhury UR, Madden BJ, Charlesworth MC, Fautsch MP. Proteome analysis of human aqueous humor. Invest Ophthalmol Vis Sci. 2010;51:4921-31.

22. Carreon T, van der Merwe E, Fellman RL, Johnstone M, Bhattacharya SK. Aqueous outflow-a continuum from trabecular meshwork to episcleral veins. Prog Retin Eye Res. 2017; 57:108-33.
23. Silverberg GD, Mayo M, Saul T, Rubenstein E, McGuire D. Alzheimer's disease, normal-pressure hydrocephalus, and senescent changes in CSF circulatory physiology: a hypothesis. Lancet Neurol. 2003;2:506-11.

24. Serot JM, Zmudka J, Jouanny P. A possible role for CSF turnover and choroid plexus in the pathogenesis of late onset Alzheimer's disease. J Alzheimers Dis. 2012;30:17-26.

25. Yoneda S, Hara H, Hirata A, Fukushima M, Inomata Y, Tanihara H. Vitreous fluid levels of beta-amyloid (1-42) and tau in patients with retinal diseases. Jpn J Ophthalmol. 2005;49:106-8. 